\section{Testicular atrophy after mumps orchitis: ultrasonographic findings}

Hyeon-Il Choi ${ }^{1}$, Dal Mo Yang ${ }^{1}$, Hyun Cheol Kim', Sang Won Kim¹, Hyun Seok Jeong', Sung Kyung Moon², Joo Won Lim²

${ }^{1}$ Department of Radiology, Kyung Hee University Hospital at Gangdong, Seoul; ' 2 Department of Radiology, Kyung Hee University Hospital, Seoul, Korea

Purpose: The purpose of this study was to describe the ultrasonographic findings of testicular atrophy after mumps orchitis.

Methods: We retrospectively reviewed the case files of eight patients (14 to 24 years old; mean, 17 years) with mumps orchitis and testicular atrophy who were treated between January 2011 and September 2017. On gray-scale and color Doppler, the ultrasonographic features of volume, shape, echogenicity, and degree of blood flow in the testes were analyzed as part of both initial and follow-up ultrasonography. The duration between the initial diagnosis of mumps orchitis and the ultrasonographic diagnosis of testicular atrophy after mumps orchitis ranged from 25 to 230 days (mean, 95.9 days).

Results: Of the eight patients with testicular atrophy after mumps orchitis, the testes were affected unilaterally in seven patients ( 6 right-sided and 1 left-sided) and bilaterally in one patient. The affected testes $(n=9)$ were $23 \%-55 \%$ (mean, $44.7 \%$ ) smaller in volume (mean, $6.3 \pm 2.0 \mathrm{~mL}$ ) than the contralateral normal testes $(n=7)$ (mean, $10.8 \pm 2.3 \mathrm{~mL}$ ) on follow-up ultrasonography $(\mathrm{P}=0.001)$. The shape of the atrophic testes was oblong in seven cases and elliptical in two cases. The atrophic testes were either heterogeneously hypoechoic with multiple hyperechoic islands $(n=7)$ or heterogeneously hyperechoic $(n=2)$. On follow-up color Doppler ultrasonography, the degree of vascularity of the atrophic testis was either similar to $(n=3)$ or lower than $(n=6)$ that of the contralateral testis.

Conclusion: On ultrasonography, atrophic testes after mumps orchitis tended to exhibit an oblong shape, heterogeneous low echogenicity with multiple hyperechoic islands, and decreased vascularity.

Keywords: Testis; Mumps; Mumps orchitis; Testicular atrophy; Ultrasonography;

Color Doppler ultrasonography

\section{Introduction}

Mumps is an acute infectious viral disease characterized by hypertrophy and pain of the salivary glands, especially the parotid glands. Although the symptoms of mumps are generally confined to infectious parotitis, severe complications such as orchitis, pancreatitis, and meningitis can occur.

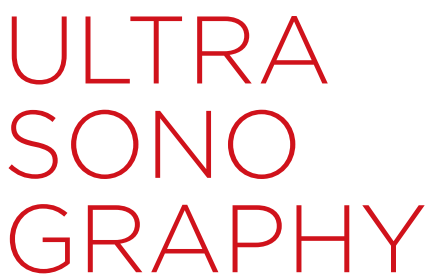

\section{ORIGINAL ARTICLE}

https://doi.org/10.14366/usg.19097 pISSN: 2288-5919 • elSSN: 2288-5943 Ultrasonography 2020;39:266-271

Received: December 31, 2019

Revised: February 25, 2020

Accepted: February 29, 2020

Correspondence to:

Dal Mo Yang, MD, Department of Radiology, Kyung Hee University Hospital at Gangdong, 892 Dongnamro, Gangdong-gu, Seoul 05278, Korea

Tel. +82-2-440-6183

Fax. +82-2-440-6932

E-mail:dmy2988@daum.net

This is an Open Access article distributed under the terms of the Creative Commons Attribution NonCommercial License (http://creativecommons.org licenses/by-nc/4.0/) which permits unrestricted noncommercial use, distribution, and reproduction in any medium, provided the original work is properly cited.

Copyright (c) 2020 Korean Society of Ultrasound in Medicine (KSUM)

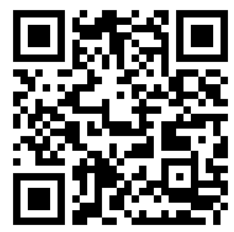

How to cite this article:

Choi HI, Yang DM, Kim HC, Kim SW, Jeong HS, Moon SK, et al. Testicular atrophy after mumps orchitis: ultrasonographic findings. Ultrasonography. 2020 Jul;39(3):266-271. 
Mumps orchitis is a complication that occurs in 15\%-30\% of adult male patients with mumps but is very rare in preadolescent patients [1]. Gray-scale ultrasonographic examination of patients with mumps orchitis shows swelling and heterogeneous hypoechogenicity of the testis along with scrotal wall thickening $[2,3]$. Color Doppler ultrasonography shows increased blood flow in the testicular parenchyma of the affected testis $[2,3]$.

The mumps virus damages the testicular tissue as a result of parenchymal edema. Increased pressure caused by parenchymal edema can induce necrosis of the seminiferous tubules and atrophy of the germinal epithelium $[4,5]$. As a result, testicular atrophy develops in $40 \%-70 \%$ of affected testicles [6].

Although several articles about testicular atrophy as a complication of mumps orchitis have been published [7-10], the associated ultrasonographic findings have not been described. The purpose of the present study is to describe the ultrasonographic findings of testicular atrophy in patients with mumps orchitis.

\section{Materials and Methods}

This retrospective study was approved by the institutional review board of our institution, and the requirement for informed consent from the patients was waived. We retrospectively reviewed the case files of eight mumps orchitis patients with testicular atrophy who were treated at Kyung Hee University Hospital at Gangdong or Kyung Hee University Hospital between January 2011 and September 2017.

Patients ranged in age from 14 to 24 years (mean, 17 years). The diagnosis of mumps orchitis in all eight patients was confirmed by the combined presence of sialadenitis and positive mumps-specific immunoglobulin $\mathrm{M}$ and/or immunoglobulin $\mathrm{G}$, with clinical findings including testicular pain, swelling, and tenderness along with ultrasonographic findings. The diagnosis of testicular atrophy was based on a testicular volume reduction of $2 \mathrm{~mL}$ or $20 \%$ compared with the contralateral normal testis [7]. The time from the initial diagnosis of mumps orchitis to the ultrasonographic diagnosis of testicular atrophy ranged from 25 to 230 days (mean, 95.9 days). In five of the eight patients, 3x1,000,000 IU of interferon alpha-2B (Intron A, Schering-Plough, Kenilworth, NJ, USA) was subcutaneously administered for 7 days.

All ultrasonographic examinations were performed with an iU22 ultrasound machine (Philips Medical Systems, Bothell, WA, USA) equipped with a 5-12 MHz linear array transducer and a LOGIQ 700MR system equipped with a $10-5 \mathrm{MHz}$ linear array transducer (GE Healthcare, Milwaukee, WI, USA). Color Doppler ultrasonography was performed with optimized color Doppler parameters. The power level, threshold, persistence, and wall filter were individually adjusted to maximize the detection of blood flow throughout the field of view. The speed scale of the color Doppler was set to the lowest detectable flow speed. The color gain control was adjusted so that background color noise was minimal or absent.

The ultrasonographic findings were determined by retrospective analysis of the images. The gray-scale and color Doppler ultrasonographic images were interpreted via the consensus of two experienced radiologists, each with more than 5 years of experience in the practice of ultrasonography.

We determined the volume, shape, echogenicity, and degree of blood flow of the testes. To measure testicular volume, the scanner was placed on the scrotum, and light pressure was exerted to avoid distorting the testicular shape. Gray-scale images of the

Table 1. Changes in testicular volume between initial US and follow-up US

\begin{tabular}{|c|c|c|c|c|c|c|}
\hline \multirow{2}{*}{ Patient No. } & \multirow{2}{*}{ Age (yr) } & \multicolumn{2}{|c|}{ Initial US } & \multicolumn{2}{|c|}{ Follow-up US } & \multirow{2}{*}{ Interval| $^{\mathrm{a})}$ (day) } \\
\hline & & Mumps (mL) & Normal (mL) & Mumps (mL) & Normal (mL) & \\
\hline 1 & 17 & 11.0 & 6.9 & 3.0 & 6.7 & 98 \\
\hline 2 & 18 & 27.2 & 14.8 & 6.7 & 10.8 & 230 \\
\hline 3 & 16 & 35 & 13.5 & 5.2 & 11.6 & 110 \\
\hline 4 & 14 & 25.4 & 11.9 & 6.2 & 13.8 & 44 \\
\hline 5 & 14 & 22.6 & 6.8 & 4.1 & 10.4 & 58 \\
\hline 6 & 18 & 19 & 10.7 & 9.9 & 12.8 & 112 \\
\hline 7 & 24 & 16.2 & 10.2 & 7.1 & 9.6 & 90 \\
\hline \multirow[t]{2}{*}{8} & 15 & 15.6 & - & 7.0 & - & 25 \\
\hline & & 11.6 & - & 7.5 & - & - \\
\hline Mean $\pm S D$ & & $20.4 \pm 7.9$ & $10.7 \pm 3.1$ & $6.3 \pm 2.0$ & $10.8 \pm 2.3$ & $95.9 \pm 62.8$ \\
\hline
\end{tabular}


Table 2. Comparison of testicular volume of NT and testes affected by $\mathrm{MO}$

\begin{tabular}{lccc}
\hline \multicolumn{1}{c}{ Group } & No. of cases & $\begin{array}{c}\text { Testicular volume, } \\
\text { mean } \pm \text { SD }(\mathrm{mL})\end{array}$ & P-value \\
\hline 1 & 7 & $10.7 \pm 3.1$ & 0.901 \\
NT initial & 7 & $10.8 \pm 2.3$ & \\
$\quad$ NT follow-up & & & \\
2 & 9 & $20.4 \pm 7.9$ & 0.001 \\
MO initial & 9 & $6.3 \pm 2.0$ & \\
MO follow-up & & $10.7 \pm 3.1$ & 0.006 \\
3 & 7 & $20.4 \pm 7.9$ & \\
NT initial & 9 & & 0.001 \\
MO initial & & & \\
4 & 7 & $6.3 \pm 2.0$ & \\
NT follow-up & 9 & & \\
MO follow-up & 9 &
\end{tabular}

NT, normal testes; MO, mumps orchitis; SD, standard deviation.

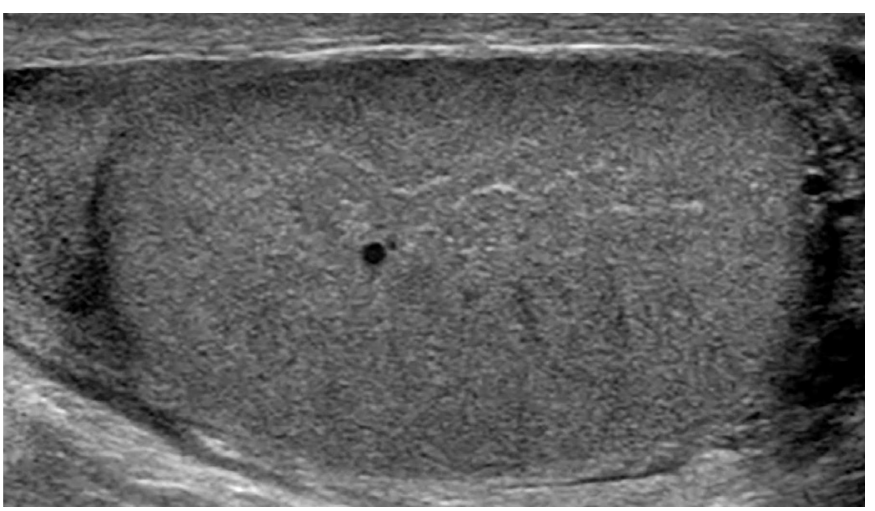

A

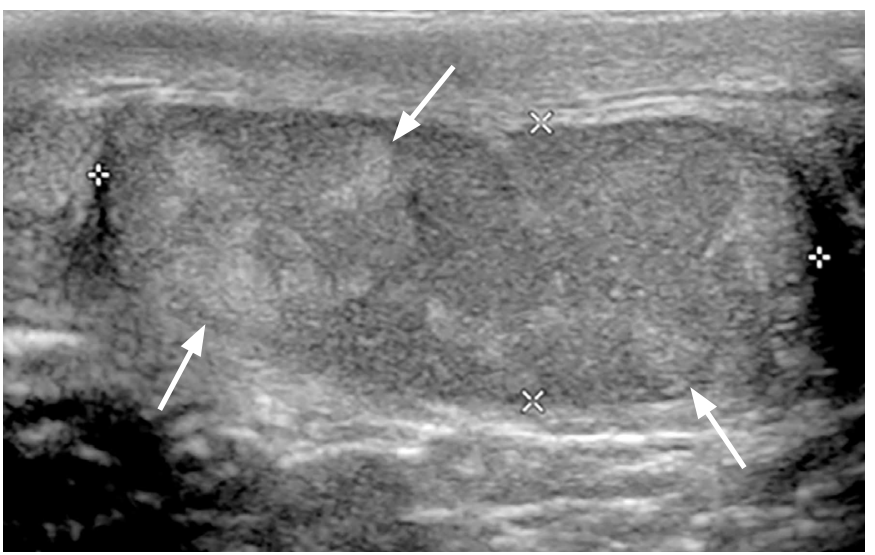

C

Fig. 1. Mumps orchitis with testicular atrophy in a 17-year-old man.

A. Longitudinal gray-scale ultrasonography shows an enlarged, hypoechoic right testis with an 11-mL testicular volume. B. Color Doppler ultrasonography shows increased blood flow in the testis. C. After 98 days, longitudinal gray-scale ultrasonography shows a testis of decreased size, with a 3-mL volume and an oblong shape. The testis was heterogeneously hypoechoic with multiple hyperechoic foci (arrows) on ultrasonography. D. Color Doppler ultrasonography shows decreased blood flow in the testis. testes were obtained in the transverse and longitudinal planes. After the maximum length, width, and height were identified on ultrasonography, the actual values were measured, and the testicular volume was calculated using the formula for an ellipsoid: volume $=\pi / 6 \times$ length $\times$ width $\times$ height [11].

We classified testicular shape as elliptical or oblong. If the testicular length was more than twice the width, we classified the shape as oblong. If the testicular length was less than twice the width, we classified it as elliptical.

The echogenicity of each testis affected by mumps orchitis was compared with that of the contralateral normal testis. The degree of blood flow to the lesion was determined through comparison with the contralateral asymptomatic side. The vascularity was classified using a 3-point scale, with 0 signifying decreased vascularity, 1 signifying similar vascularity, and 2 signifying increased vascularity compared with the contralateral normal testis. In the single case of bilateral involvement of mumps orchitis, the echogenicity and blood
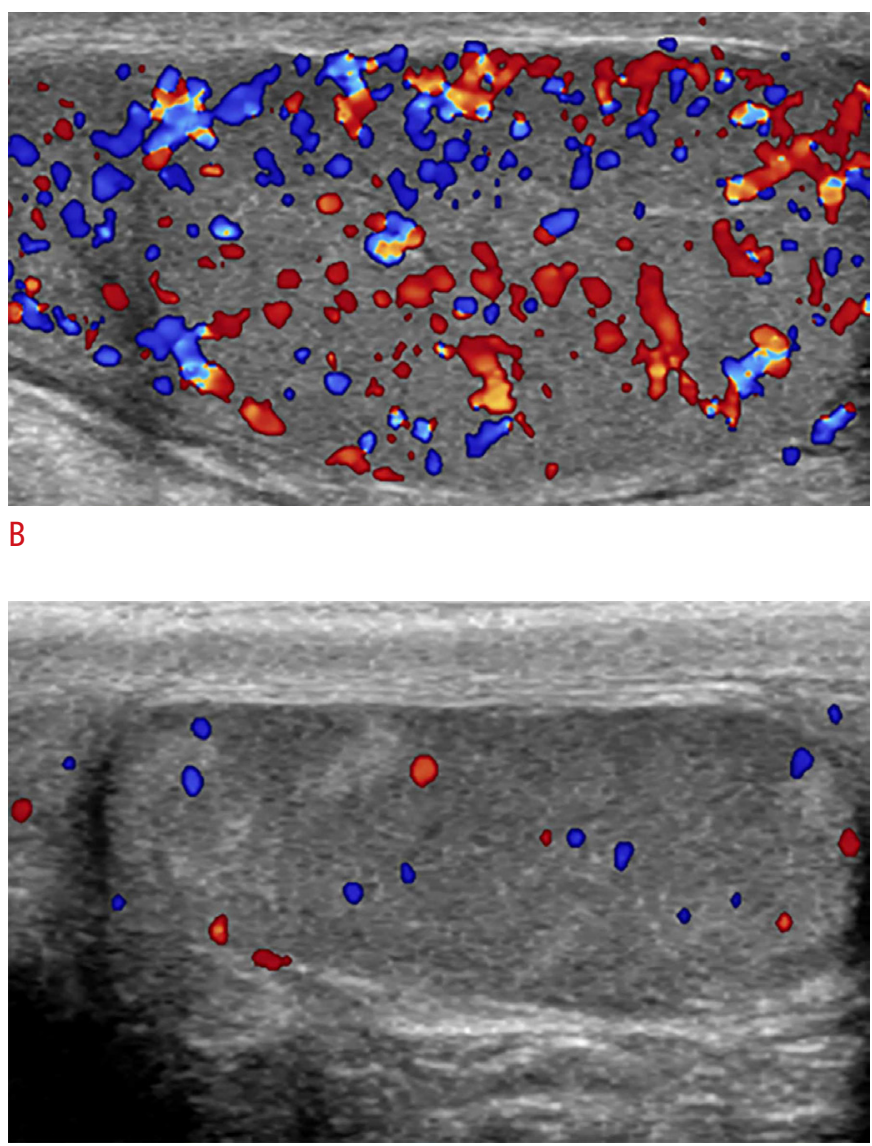

D 


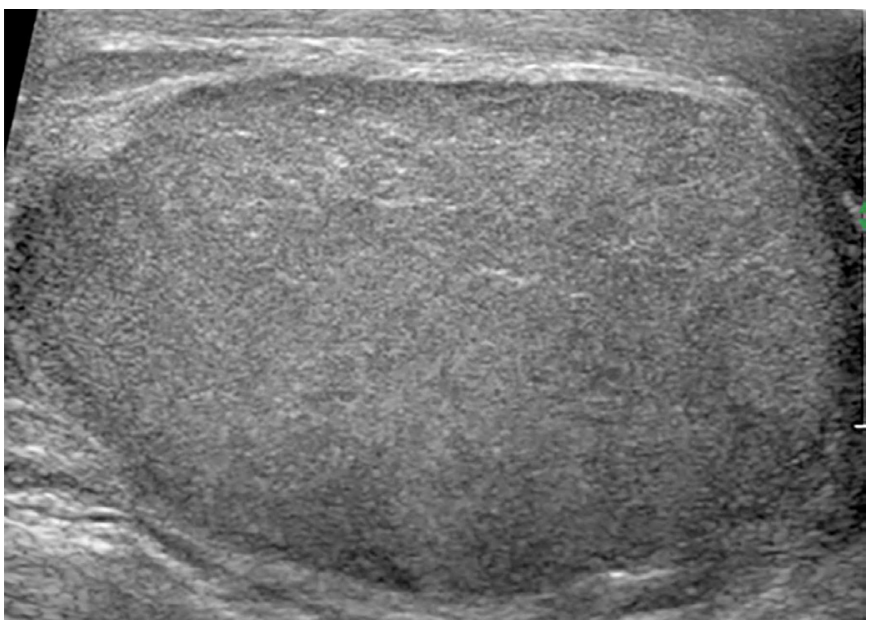

A

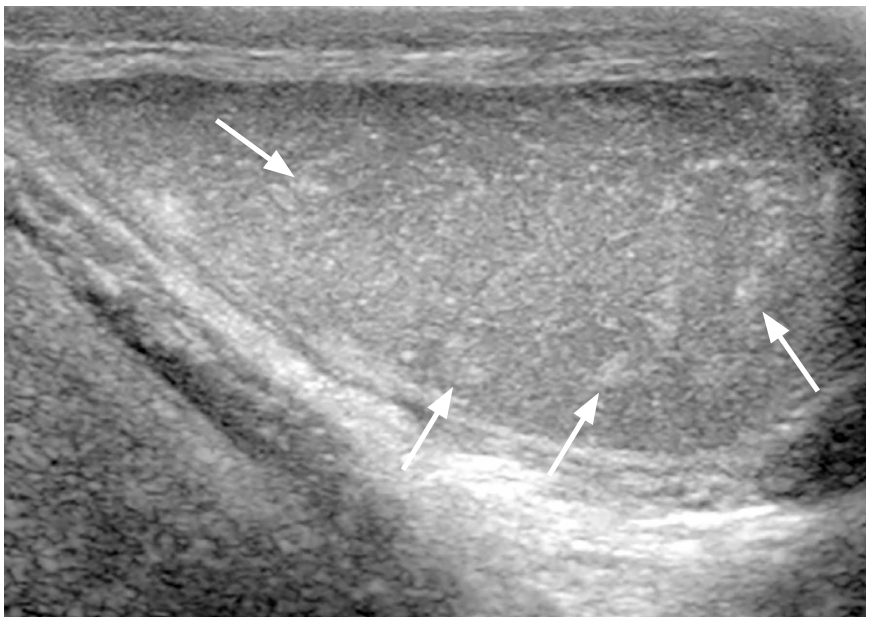

C

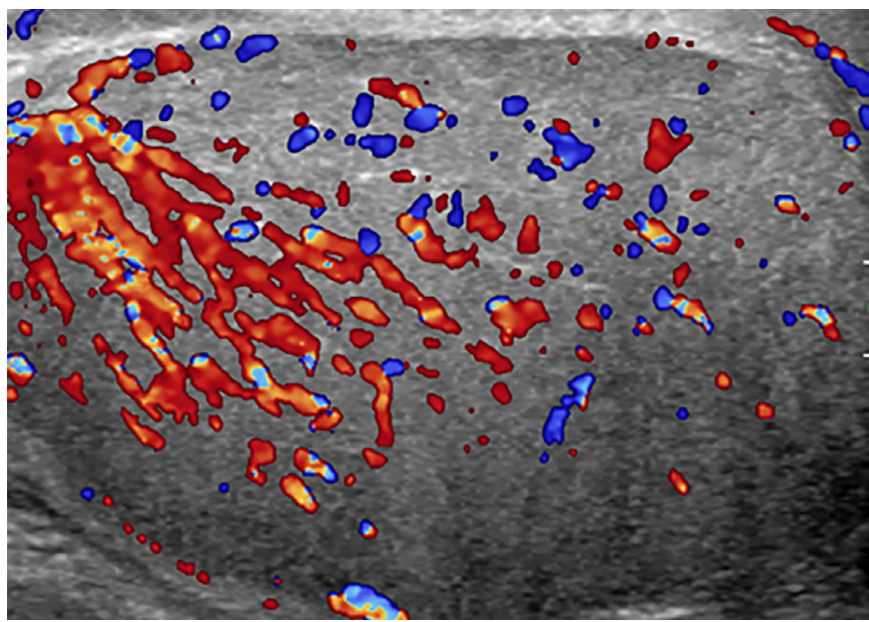

B

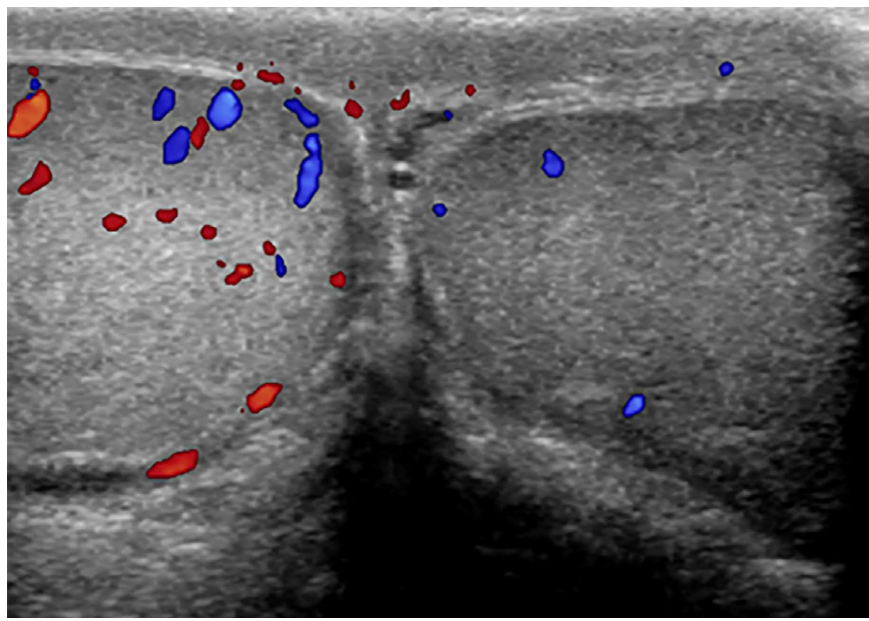

D

Fig. 2. Mumps orchitis with testicular atrophy in an 18-year-old man.

A. Longitudinal gray-scale ultrasonography shows an enlarged, heterogeneously hypoechoic left testis with a 27.2-mL testicular volume. B. Color Doppler ultrasonography shows increased blood flow in the testis. C. After 230 days, longitudinal gray-scale ultrasonography shows a testis of decreased size, with a $6.7-\mathrm{mL}$ volume and an oblong shape. The testis was heterogeneously hypoechoic with multiple hyperechoic foci (arrows) on ultrasonography. D. Color Doppler ultrasonography shows decreased blood flow in the affected left testis compared with the right testis.

flow were compared with the contralateral normal testes of the seven other patients.

All data were collected and analyzed with SPSS version 18.0 (SPSS Inc., Chicago, IL, USA). The independent t test was used to evaluate the differences in age and volume. A P-value of less than 0.05 was considered to indicate statistical significance.

\section{Results}

Testes were affected unilaterally in seven patients ( 6 right-sided and 1 left-sided) and bilaterally in one patient. The mean testicular volume at the initial ultrasonographic examination was $20.4 \pm 7.9$
$\mathrm{mL}$ (range, 11.6 to $27.2 \mathrm{~mL}$ ) in the testes affected by mumps orchitis and $10.7 \pm 3.1 \mathrm{~mL}$ (range, 6.8 to $14.8 \mathrm{~mL}$ ) in the unaffected testes (Table 1). The mean testicular volume at the follow-up ultrasonographic examination was $6.3 \pm 2.0 \mathrm{~mL}$ (range, 3.0 to 10.3 $\mathrm{mL}$ ) for the affected testes and $10.8 \pm 2.3 \mathrm{~mL}$ (range, 6.7 to $13.8 \mathrm{~mL}$ ) for the unaffected testes (Table 1).

On initial ultrasonography, all nine affected testes had larger volumes than the corresponding contralateral normal testes $(n=7$, $\mathrm{P}=0.006$ ) (Table 2). However, on follow-up ultrasonography, the testicular volumes of the testes affected by mumps orchitis were smaller than those of the contralateral normal testes $(n=7)$ by $23 \%$ $55 \%$ (mean, 44.7\%; $P=0.001$ ) (Figs. 1, 2). Compared to the initial 
Table 3. Summary of ultrasonographic (US) findings of testicular atrophy in mumps orchitis

\begin{tabular}{|c|c|c|c|c|c|c|c|c|}
\hline \multirow{2}{*}{$\begin{array}{c}\text { Patient } \\
\text { No. }\end{array}$} & \multirow{2}{*}{$\begin{array}{c}\text { Age } \\
(y r)\end{array}$} & \multirow{2}{*}{ Location } & \multicolumn{3}{|c|}{ Initial US } & \multicolumn{3}{|c|}{ Follow-up US } \\
\hline & & & Shape & Echogenicity & Vascularity grade & Shape & Echogenicity & Vascularity grade \\
\hline 1 & 17 & Right & Elliptical & Hypoechoic & 2 & Oblong & Heterogeneously hypoechoic & 0 \\
\hline 2 & 18 & Left & Elliptical & Heterogeneously hypoechoic & 2 & Oblong & Heterogeneously hypoechoic & 0 \\
\hline 3 & 16 & Right & Elliptical & Hypoechoic & 2 & Oblong & Heterogeneously hypoechoic & 0 \\
\hline 4 & 14 & Right & Elliptical & Hypoechoic & 2 & Oblong & Heterogeneously hypoechoic & 0 \\
\hline 5 & 14 & Right & Elliptical & Heterogeneously hypoechoic & 2 & Oblong & Heterogeneously hypoechoic & 0 \\
\hline 6 & 18 & Right & Elliptical & Hypoechoic & 2 & Elliptical & Heterogeneously hyperechoic & 1 \\
\hline 7 & 24 & Right & Elliptical & Hypoechoic & 2 & Elliptical & Heterogeneously hyperechoic & 0 \\
\hline \multirow[t]{2}{*}{8} & 15 & Right & Elliptical & Hypoechoic & 2 & Oblong & Heterogeneously hypoechoic & 1 \\
\hline & & Left & Elliptical & Hypoechoic & 2 & Oblong & Heterogeneously hypoechoic & 1 \\
\hline
\end{tabular}

Vascularity was graded using a 3-point scale: 0, decreased vascularity compared with the contralateral normal testis; 1, similar vascularity to the contralateral normal testis; and 2, increased vascularity compared with the contralateral normal testis.

testicular volumes, the volumes of the affected testes were reduced by $35 \%-92 \%$ (mean, $68.3 \%$ ) on follow-up ultrasonography $(n=9)$. The affected testes exhibited a significant difference in volume between initial ultrasonography and follow-up ultrasonography $(\mathrm{P}=0.001)$.

In terms of testicular shape, all nine testes impacted by mumps orchitis were elliptical on initial ultrasonography (Table 3). On follow-up ultrasonography, however, the testicular shape had changed to oblong in seven testes (Figs. 1, 2).

In terms of echogenicity, testes affected by mumps orchitis appeared hypoechoic $(n=7)$ or heterogeneously hypoechoic $(n=2)$ on initial ultrasonography and heterogeneously hypoechoic with multiple hyperechoic islands $(n=7)$ or heterogeneously hyperechoic $(n=2)$ on follow-up ultrasonography (Table 3, Figs. 1, 2). On color Doppler ultrasonography, the degree of blood flow was relatively high (grade 2) in all nine cases at the initial examination. On followup color Doppler ultrasonography (Table 3 ), the degree of blood flow was grade $1(n=3)$ or grade 0 ( $n=6)$ (Figs. 1, 2).

\section{Discussion}

Testicular atrophy is a reduction in testicular size that can occur after epididymo-orchitis, testicular torsion, unilateral cryptorchidism, varicocele, or trauma [12]. Lee et al. [7] stated that the criterion for testicular atrophy is a testicular volume reduction of $2 \mathrm{~mL}$ or $20 \%$ compared with the contralateral normal testis. In our study, after mumps orchitis $(n=9)$, atrophic testes had volumes $23 \%-55 \%$ (mean, $44.7 \%$ ) smaller than the contralateral normal testes $(n=7)$.

The pathogenesis of testicular atrophy in mumps orchitis is as follows. Infection causes edema or congestion, as well as perivascular and interstitial infiltration of lymphocytes. The increased pressure on the seminiferous tubules leads to necrosis due to the barrier effect of the tunica albuginea of the testis $[4,5]$. Then, spermatogenesis stops, and necrotic cells, debris, and phagocytes accumulate in the seminiferous tubules [4]. Finally, seminiferous tubule necrosis leads to hyalinization, fibrosis, and atrophy of the testes [8].

In terms of testicular shape, in seven cases of mumps orchitis in the present study, the shape became more slender (changing from elliptical to oblong) between initial and follow-up ultrasonography. Therefore, we suggest that the testicular volume loss in mumps orchitis is more severe in the axial dimension than in the longitudinal dimension.

Regarding echogenicity, most of the atrophic testes in our study appeared heterogeneously hypoechoic with multiple hyperechoic islands on follow-up ultrasonography $(n=7)$. A previous study found that, in cases of testicular atrophy after trauma, two of five patients showed heterogeneous echogenicity of the affected testis on ultrasonography [12]. Heterogeneous echogenicity of the atrophic testes is present due to tubular atrophy and fibrous thickening of the walls of the seminiferous tubules [13]. The hypoechoic area of the heterogeneous testis corresponds to tubular atrophy and sclerosis [13]. Interestingly, in all but one of the cases in the present study, multiple small irregular hyperechoic foci were scattered throughout the affected hypoechoic atrophic testes. Although we could not conclude that a pathologic correlation was present, our results suggest that the hyperechoic islands may be remnants of normal testicular parenchyma.

On color Doppler ultrasonography, most cases of mumps orchitis show hypervascularity in the testicular parenchyma [3]. However, in a previous study, the vascularity of testes with testicular atrophy was decreased in $40 \%$ of cases [12]. In our study, six of the nine testes $(67 \%)$ showed decreased vascularity compared to the contralateral 
normal testis. The decreased vascularity observed in cases of testicular atrophy is related to tubular atrophy and tubular sclerosis.

In mumps orchitis cases, detection of testicular atrophy using ultrasonography is important because male infertility develops in $37 \%-87 \%$ of patients with testicular atrophy [9]. Testicular atrophy is seen in $40 \%-70 \%$ of mumps orchitis cases [6]. Testicular volume has been found to be correlated with parameters of testicular function including sperm density, total sperm count, total mobile sperm count, motility percentage, serum follicle-stimulating hormone level, and luteinizing hormone level [14]. Spermatogenesis may be disrupted in $50 \%$ of patients at 1 to 3 months after recovery from both unilateral and bilateral mumps orchitis [15]. Oligospermia is seen in $7 \%-13 \%$ of mumps orchitis cases [6], and bilateral mumps orchitis causes irreversible infertility in $30 \%-87 \%$ of cases [8].

Preventing testicular atrophy is important in patients with mumps orchitis. Creating an early incision in the tunica albuginea to reduce intratesticular pressure may assist in avoiding testicular atrophy. Erpenbach [9] found that interferon alpha-2B prevents testicular atrophy in cases of mumps orchitis via a direct antiviral effect in which transcriptase-induced viral replication is interrupted. However, other studies have found that interferon alpha-2B is not effective in preventing testicular atrophy in mumps orchitis cases $[8,10]$.

Our study was limited by its retrospective design and small study population. Therefore, further studies with larger sample sizes are necessary. In addition, we could not determine the nature of the hyperechoic foci within the testicular parenchyma on ultrasonography because we were unable to determine whether a pathologic correlation was present.

In conclusion, ultrasonography may be helpful in the diagnosis of testicular atrophy after mumps orchitis by enabling the detection of the features typical to this condition, namely small testis with an oblong shape, heterogeneous low echogenicity with multiple hyperechoic islands, and decreased vascularity.

ORCID: Hyeon-II Choi: https://orcid.org/0000-0002-8739-7965; Dal Mo Yang: https:// orcid.org/0000-0001-7766-5826; Hyun Cheol Kim: https://orcid.org/0000-00023121-092X; Sang Won Kim: https://orcid.org/0000-0002-1999-9163; Hyun Seok Jeong: https://orcid.org/0000-0003-0229-2257; Sung Kyung Moon: https://orcid.org/00000003-4831-3439; Joo Won Lim: https://orcid.org/0000-0002-7989-3408

\section{Author Contributions}

Conceptualization: Yang DM. Data acquisition: Choi HI, Yang DM, Kim HC, Jeong HS, Moon SK, Lim JW. Data analysis or interpretation: Yang DM, Kim SW. Drafting of the manuscript: Yang DM, Kim HC, Kim SW, Choi HI, Jeong HS, Moon SK. Critical revision of the manuscript: Yang DM, Lim JW. Approval of the final version of the manuscript: all authors.

\section{Conflict of Interest}

No potential conflict of interest relevant to this article was reported.

\section{References}

1. Hviid A, Rubin S, Muhlemann K. Mumps. Lancet 2008;371:932944.

2. Park SJ, Kim HC, Lim JW, Moon SK, Ahn SE. Distribution of epididymal involvement in mumps epididymo-orchitis. J Ultrasound Med 2015;34:1083-1089.

3. Tarantino L, Giorgio A, de Stefano G, Farella N. Echo color Doppler findings in postpubertal mumps epididymo-orchitis. J Ultrasound Med 2001;20:1189-1195.

4. Gall EA. The histopathology of acute mumps orchitis. Am J Pathol 1947;23:637-651.

5. Charny CW, Meranze DR. Pathology of mumps orchitis. J Urol 1948;60:140-146.

6. Singh $R$, Mostafid $H$, Hindley RG. Measles, mumps and rubella: the urologist's perspective. Int J Clin Pract 2006;60:335-339.

7. Lee JW, Lee TY, Choi HY. Change of the testicular volume after mumps orchitis. Korean J Urol 2007;48:542-547.

8. Yeniyol CO, Sorguc S, Minareci S, Ayder AR. Role of interferonalpha-2B in prevention of testicular atrophy with unilateral mumps orchitis. Urology 2000;55:931-933.

9. Erpenbach KH. Systemic treatment with interferon-alpha 2B: an effective method to prevent sterility after bilateral mumps orchitis. J Urol 1991;146:54-56.

10. Yapanoglu T, Kocaturk H, Aksoy Y, Alper F, Ozbey I. Long-term efficacy and safety of interferon-alpha-2B in patients with mumps orchitis. Int Urol Nephrol 2010;42:867-871.

11. Paltiel HJ, Diamond DA, Di Canzio J, Zurakowski D, Borer JG, Atala A. Testicular volume: comparison of orchidometer and US measurements in dogs. Radiology 2002;222:114-119.

12. Cross JJ, Berman LH, Elliott PG, Irving S. Scrotal trauma: a cause of testicular atrophy. Clin Radiol 1999;54:317-320.

13. Harris RD, Chouteau C, Partrick M, Schned A. Prevalence and significance of heterogeneous testes revealed on sonography: ex vivo sonographic-pathologic correlation. AJR Am J Roentgenol 2000;175:347-352.

14. Sakamoto $H$, Ogawa $Y$, Yoshida $H$. Relationship between testicular volume and testicular function: comparison of the Prader orchidometric and ultrasonographic measurements in patients with infertility. Asian J Androl 2008;10:319-324.

15. Bartak V. Sperm count, morphology and motility after unilateral mumps orchitis. J Reprod Fertil 1973;32:491-494. 